\title{
Millimeter wave therapy for abdominal incision wound: A clinical study
}

\author{
Meena Pradhan', Shao Yong ${ }^{1}$, Luo Yun Peng ${ }^{2}$ \\ ${ }^{1}$ Department of Obstetrics and Gynecology, ${ }^{2}$ Department of Pathophysiology, The First Affiliated Hospital of Chongqing Medical University, \\ Chongqing 40016, PR China
}

\section{A B S T R A C T}

Background: This case-control study, conducted in 2012, was performed to determine if MMWT, a non invasive therapeutic treatment modality, could reduce postoperative pain and infection in patients who had abdominal sections. Methods: The study group (30 patients) incision sites were exposed to MMWT within 24 hour of cesarean and the control group (90 patients) was not exposed to MMWT. Millimeter wave apparatus with intensity of $38 \mathrm{GHz}$ and power flux output density of $40 \mathrm{~mW} / \mathrm{cm}^{2}$ was used for subjecting the cesarean incision site to MMWT. The first exposure to MMWT was done for 30 minutes within 24 hour of cesarean section. In total, they were exposed for 30 minutes daily for 3 days. Wound healing was assessed every day. Results: Pain intensity score in the exposure group reduced significantly compared to the control group. The mean pain intensity score of the study group on the 3 rd postoperative day was $3.3 \pm 1.8(95 \% \mathrm{Cl}$ 2.6-3.9) compared to $4.2 \pm 1.6(95 \% \mathrm{Cl} 3.8-4.5)$ of the control group $(\mathrm{P}<0.01)$. Pain reduction rate on day 3 was significantly higher than day 1 in the study group compared to the control group ( $P=0.0024)$. Routine white blood cell (WBC) count was significantly lower in the MMWT treated patients $\left(11.3 \pm 1.8 \times 10^{9} ; 95 \% \mathrm{Cl} 10.6-12\right)$ compared to the control group $\left(12.3 \pm 2.9 \times 10^{9} ; 95 \% \mathrm{Cl} 11.7-12.9\right)(\mathrm{P}<0.03)$. Conclusions: The results of our study verified that MMWT can relieve post operative pain, decrease post operative morbidities, enhance early mobilization, and can serve as an alternative tool to suppress pain and cure infection.

Key words: Cesarean Section, Millimeter Wave Therapy, Pain Relief, Wound infection

\section{INTRODUCTION}

Millimeter wave therapy (MMWT), first developed in Soviet Union 40 years ago, is an apparatus, broadly used for therapeutic purpose in the treatment of many diseases including bronchial asthma, diabetic neuropathies, peptic ulcer, gastric polyps, infantile cerebral paralysis and postoperative pain relief effectively. ${ }^{1}$ In a double blinded randomized control trial study, MMWT effectively suppressed pain to cold stimulus in healthy human body. ${ }^{2}$ Millimeter waves penetrate less than $1 \mathrm{~mm}$ below the surface layers of the skin. If the intensity of MMWT is less than $20 \mathrm{~mW} / \mathrm{cm}^{2}$, there is no perceptible heat production. At $40 \mathrm{~mW} / \mathrm{cm}^{2}$, some subjects will sense a temperature increase. $^{3}$
Post cesarean infection (PCI) involving infection of the skin and subcutaneous tissue at the surgical incision site is typically caused by skin flora, such as Staphylococcus $s p$., Streptococcus sp., or mixed aerobic/anaerobic bacteria. They observed that $8.1 \%$ of post cesarean wounds were infected, and $42 \%$ of the infections were due to staphaureus, and $86.9 \%$ due to other microorganism. ${ }^{4}$ MMWT successfully treated skin diseases, such as psoriasis and atopic dermatitis by stimulating tissue's ability to repair and regenerate. ${ }^{5}$ The low intensity wave at incision site is believed to be absorbed by human tissue to stimulate opioids in the whole body to reduce postoperative pain. ${ }^{6}$ Although there is an increasing trend of women choosing cesarean delivery as safe delivery method, it often leads to operation induced lower abdomen pain, incision site infection, poor 
wound healing, incision hernia, permanent lower abdomen scar, and increased risk of morbidity. Normally, patients are discharged within 3-4th postoperative day after wound is recovered. However, if the wound is not properly healed and exudation continues from incision site, the hospital stay will be prolonged or frequent visit to hospital for regular dressing would be necessary for postpartum mothers. Thus, effective pain management is an essential responsibility of postoperative management. The purposes of this study was to effect proper wound healing and reduce post operative pain and morbidities in patients who had cesarean sections, by using MMWT.

\section{MATERIAL AND METHODS}

We used Millimeter wave apparatus (Model no. HB/H-a. Serial No 120213) manufactured by Hengbo Medical Instrument Company, Cheng Du, China, with power flux density of $40 \mathrm{~mW} / \mathrm{cm}^{2}$, and frequency output of $38 \mathrm{GHz}$. The surface of incision site was exposed to MMWT for 30 minutes each for 3 consecutive days. Incision site was observed on each day before and after MMWT exposure. The dressing pad of the incision site was removed before MMW'T exposure to ensure direct contact of the millimeter wave with the incision site. The incision site was covered by a new dressing pad after MMWT exposure. Routine alternate day dressing was done with sterile betadine soaked gauze on incision site of both the case- control groups.

To minimize bias, the control group was randomly chosen from women who underwent cesarean section (CS) during the same study period as the MMWT exposure cases. A total of 120 cases of post cesarean patients who had undergone CS during 1st January - 31st December 2012, were randomly selected for the study. After cesarean section, all the patients were treated with routine intravenous analgesia and sedative drugs (Tramadol $100 \mathrm{mg}$ (8 vial) + Nefopam Hydrochloride $20 \mathrm{mg}$ (5 vial)+ Dexamethasone $5 \mathrm{mg}+$ normal saline $80 \mathrm{ml}$ ) through patient controlled intravenous analgesia pump (PCIA) from Cesarean Medical Electronics, Lichtenstein, Germany. The PCIA was attached to intravenous line with a basal infusion rate of $1 \mathrm{ml}$ per hour (maximum dose of up to $5 \mathrm{ml}$ per hour) for 24 hours postoperatively. The study group included patients, who were normal healthy pregnant, gestational diabetes mellitus, premature rupture of membrane, and placenta previa, and exposed to MMWT ( $\mathrm{n}=30)$ within $24 \mathrm{~h}$ of CS and the control group ( $n=90)$ were not exposed to MMWT. The Ethics Committee at the first affiliated hospital of Chongqing Medical University, Chongqing, China, approved the study. Patients with chorioamnitis, any systemic or local infectious disease diagnosed prior CS, MMW'T exposure after $>24 \mathrm{~h}$ of cesarean section, severe postpartum hemorrhage, emergency peripartum hysterectomy, pregnancy induced hypertension (pre-eclampsia), were excluded from the study. The response of the patients to nociceptive stimulus was assessed in terms of pain threshold numerical values: no pain 0 , mild pain 2 , moderate pain 4 , distinct pain 6 , severe pain 8 and extreme pain 10. Pain was assessed everyday before and after MMW'T exposure.

For routine white blood cell (WBC) count, neutrophil and lymphocyte percentage, $1 \mathrm{ml}$ of maternal blood was drawn in ethylenediaaminetetraacetic acid (EDTA) tube. WBC count was measured using an automated hematology Analyzer, (Model no XE-500, Serial number A2647, Manufacture KOBE, Sysmex co-operation, Japan). The results of preoperative routine blood examination $(24 \mathrm{~h}$ before CS) and postoperative (after $48 \mathrm{~h}$ ) routine blood examination for study and especially white blood cell count $(\mathrm{WBC})$, neutrophil $(\mathrm{N})$ and lymphocyte $(\mathrm{L})$ percentage, were selected for accurate recognition of infection or inflammation as these marker are considered valid, cost effective and accessible. Immediate and daily post CS routine blood test for limited criteria were excluded from our study. The incision site in both the groups was carefully observed everyday for possible inflammatory reaction, such as proper wound healing, erythema or edema and production of exudate fluids. Before MMWT exposure, all the patients were explained about its effects and benefits and written consent were taken from the study group for clinical analysis. As patients and their family feared that MMW'T exposure might cause harm to them, we explained to them that there is no adverse effect or harm to mother or its surrounding. After exposure to MMWT, its efficacy was assessed by pain scoring interview next day, and monitoring sleeping pattern until discharge from hospital.

The data obtained was statistically analyzed using SAS version 8. Comparison between the two groups was done using student's $t$ test, and Wilcoxon test for pain reduction score. $\mathrm{p}<0.05$ was considered statistically significant.

\section{RESULTS}

Most patients answered MMWT was effective treatment equipment for post operative pain physiologically and psychologically. Table 1 shows clinical evaluation and general characteristic of postoperative pain. Postoperative pain assessment showed no significant difference ( $p>0.05)$ on day one and day two of MMWT exposure; however, pain intensity was significantly reduced in day three of exposure to MMWT group. The pain score of study group on day three was $3.3 \pm 1.895 \%$ CI (2.6-3.9), categorized as mild to moderate pain score compared to control 
group 4.2 $\pm 1.6(95 \%$ CI 3.8-4.5), categorized as moderate to distinct pain ( $\mathrm{p}<0.05)$.

Table 2 shows a decrease in the pain reduction score over three days, for repeated measurement of every case. Wilcoxon test for pain reduction rate from day one to day three is higher in MMWT group (Mean \pm SD) is $54.32 \pm 25.87$ compared to the control $29.31 \pm 51$ $(\mathrm{p}=0.0024)$. With 25 th percentile the pain reduction rate is $37.5 \%$ in the study group. In 50th percentile, pain reduction rate was $60 \%$ in study group and $47.2 \%$ in control group. We observed significant reduction of pain between treatment group and control group.

Table 3 shows the results of physical examination of abdomen, incision site observation and other assessment postoperatively. In exposed group, erythema at incision site occurred in two and edema in one patient, but resolved completely in third day before discharge from hospital. The patients had good sleep during their hospital stay. MMWT exposure was found to sedate patients with psychological trauma of operation. However, in control group, sleep

\begin{tabular}{|c|c|c|c|}
\hline Variable & $\begin{array}{c}\text { Cases }(n=30) \\
\text { mean } \pm \text { SD } \\
(95 \% \mathrm{Cl})\end{array}$ & $\begin{array}{c}\text { Control }(n=90) \\
\text { mean } \pm S D \\
(95 \% \mathrm{Cl})\end{array}$ & $P$ value \\
\hline Age (years $\pm S D$ ) & $36 \pm 5.4$ & $35.4 \pm 4.8$ & - \\
\hline Weeks of gestation & $36.7 \pm 3.8$ & $37.8 \pm 3.0$ & - \\
\hline Day 1 & $7.4 \pm 1.3(6.9-7.8)$ & $6.8 \pm 7.2(6.3-7.2)$ & 0.07 \\
\hline Day 2 & $5.3 \pm 1.8(4.6-5.9)$ & $5.6 \pm 2.1(5.2-6.1)$ & 0.4 \\
\hline Day 3 & $3.3 \pm 1.8(2.6-3.9)$ & $4.2 \pm 1.6(3.8-4.5)$ & 0.01 \\
\hline
\end{tabular}

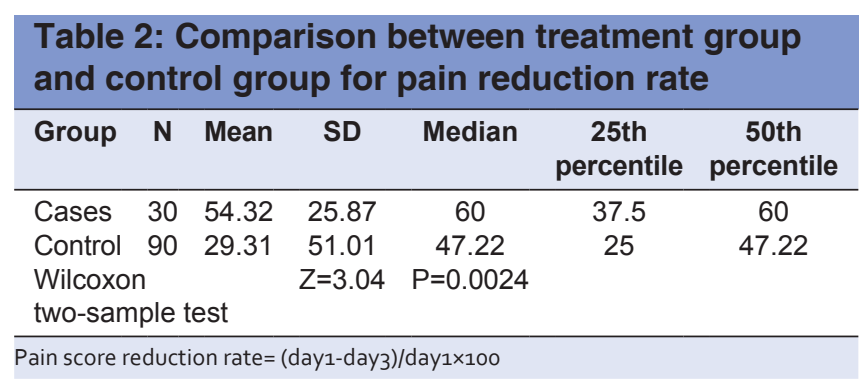

Table 3: Comparison between treatment group vs. control group for postoperative characteristic of cesarean incision wound

\begin{tabular}{lcc}
\hline Variable & Cases $\mathbf{n = 3 0 ~ ( \% ) ~}$ & Control $\mathbf{n = 9 0 ~ ( \% ) ~}$ \\
\hline Wound exudation & 0 & $12(13.3 \%)$ \\
Erythema & $2(6.6 \%)$ & $11(12.2 \%)$ \\
Edema & $1(3.3 \%)$ & $5(5.5 \%)$ \\
Abdomen distension & 0 & $7(7.7 \%)$ \\
Wound gap & 0 & $2(2.2 \%)$ \\
Re stitch & 0 & $1(1.1 \%)$ \\
Sleep deprivation & 0 & $49(54.4 \%)$ \\
Difficult micturation & 0 & $1(1.1 \%)$ \\
\hline
\end{tabular}

deprivation occurred in 49/90 (54.4\%), wound exudation in $12 / 90(13.3 \%)$, erythema in $11 / 90(12.2 \%)$, skin closure site edema in $5 / 90(5.5 \%)$, abdomen distension in $7 / 90(7.7 \%)$, wound gap in $2 / 90(2.2 \%)$, re stitch required in $1 / 90(1.1 \%)$, and difficult micturation in $1 / 90(1.1 \%)$ cases. They were all treated with routine dressing and continued follow up in outpatient based dressing until the wound was healed.

Table 4 shows the results of both preoperative and postoperative blood routine examination including white blood cell (WBC) count, neutrophil (N\%) and lymphocyte $(\mathrm{L} \%)$ for both the MMWT exposure group and the control group. Preoperative WBC count, neutrophil (N\%), and lymphocyte $(\mathrm{L} \%)$ was statistically not significant ( $\mathrm{P}>0.05)$. On the other hand, postoperative WBC count between study group $\left(11.3 \pm 1.8 \times 10^{9} ; 95 \%\right.$ CI 10.6-12) and control group $\left(12.3 \pm 2.9 \times 10^{9} / 1 ; 95 \%\right.$ CI $\left.11.7-12.9\right)$ was statistically significant $(\mathrm{P}<0.05)$. Significantly lowered WBC in the MMWT study group compared to the control group suggests that MMWT has anti-inflammatory action on human body. A WBC count below $4.0 \times 10^{9} / 1$ or above $12.0 \times 10^{9} / 1$ is determined considered as systemic inflammatory response. ${ }^{7}$ However, neutrophil (N\%), and lymphocyte $(\mathrm{L} \%)$ were statistically not significant $(\mathrm{P}>0.05)$.

\section{DISCUSSION}

Absorption of physical wave radiation by skin surface during MMWT exposure results in occurrence of chemical reaction within specific cells and tissues, and the whole body reacts positively by changing functional behavior after single exposure of MMWT. Although the exact mechanism of MMWT on cells is not known yet, the clinical therapeutic application is versatile. Several published reports suggest that the action of MMWT on inflammation is by improving microcirculation in injured tissue and stimulating physiological and reparative regeneration, ${ }^{8-10}$ increasing proliferative activity of fibroblasts along with improvement of the immune function. ${ }^{11}$

Some edema and inflammation in a wound is necessary prerequisite for proper healing, because inflammation is the initial phase of wound healing. Changes in blood flow are responsible for the clinical symptoms used to identify an inflammatory response. Increased level of WBC count observed in post cesarean delivery may be because of local tissue damage. Significantly reduced level of white blood cell (WBC) count in MMWT treated group on 2nd postoperative day compared to the control group indicates that MMWT has significant role in controlling infection by strengthening immune function. All the cesareans delivery women were treated with intravenous 


\begin{tabular}{|c|c|c|c|}
\hline Variable & $\begin{array}{c}\text { Cases }(n=30) \\
\text { mean } \pm S D(95 \% \mathrm{Cl})\end{array}$ & $\begin{array}{c}\text { Control }(n=90) \\
\text { mean } \pm S D(95 \% \mathrm{Cl})\end{array}$ & $P$ value \\
\hline Pre operative WBC & $8.4 \pm 2.3 \times 10^{9}(7.5-9.4)$ & $9.1 \pm 2.5 \times 10^{9}(8.6-9.6)$ & 0.2 \\
\hline Pre operative Neutrophil \% & $74.2 \pm 5.6(72.2-76.4)$ & $76.2 \pm 5.5(75.1-77.4)$ & 0.1 \\
\hline Pre operative Lymphocytes \% & $19.2 \pm 4.9(17.4-20.1)$ & $17.7 \pm 4.8(16.6-18.7)$ & 0.1 \\
\hline Post operative WBC & $11.3 \pm 1.8 \times 10^{9}(10.6-12)$ & $12.3 \pm 2.9 \times 10^{9}(11.7-12.9)$ & 0.03 \\
\hline Post operative Neutrophil \% & $80.6 \pm 4.9(78.7-82.4)$ & $81.7 \pm 5.6(80.5-82.9)$ & 0.3 \\
\hline Post operative Lymphocyte \% & $14.5 \pm 4.8(12.7-16.3)$ & $13.3 \pm 4.0(12.5-14.2)$ & 0.2 \\
\hline
\end{tabular}

antibiotic for 2 days during hospital stay and further three days were completed with oral antibiotics. Approximately $50-70 \%$ of WBC is neutrophil. Damage and inflammation of tissue can lead to high neutrophil count. Lymphocyte count decreased in immunocompromised, sepsis patients resulting in poor wound healing. ${ }^{12}$ We hypothesize that activation of lymphocyte subset $T$ helper cell in the body is responsible for tissue repair and regeneration. After exposure to MMW'T, $\mathrm{T}$ helper cell's subset $\mathrm{T}$ helper1 (Th1) functional cell gets stimulation and produce opioids resulting in decreased pain. Another hypothesis is that MMWT radiation therapy further communicates with $T$ helper 1 to stimulate increased level of interferon gamma (INF- $\gamma$ ), interleukin 2 (IL-2), monocyte and ultimately macrophage that engulf debris of cells and tissue of incised wound and improve wound healing. ${ }^{13}$ This is natural phenomenon of wound healing; however MMW'T exposure would further enhance ability of healing function by promoting immunity of these cells.

Another reason behind pain relief by MMWT exposure is that electromagnetic signals are absorbed by receptors of the dermis of free nerve endings with thin myelinated or unmyelinated nerve fibers. These sensory nerve endings gather information of potential effect of wave radiation and send it to the brain. MMWT helps to reduce the microcapillary circulation to the surface of the skin resulting in reduction in erythema that may lessen the buildup of edema and swelling. Pain was significantly reduced when wounds was exposed to MMWT for 3 days $(p<0.05)$. This assumption may arise from convergence of nociceptive efferent fibers from dermatomes of internal organs to the same neurons of pain pathways. This gives rise to skin hypersensitivity because visceral impulses raise the excitation of intercalary neurons, which leads to pain relief. $^{9}$

It is important to assess the post operative pain, wound healing for the procedure in light of the patient's general health status, the threshold of pain, and the likelihood that it can reduce pain. The postoperative pain affects normal body movement, irritation and sleep deprivation.
Intramuscular administration of analgesic drugs for post-operative pain control is generally avoided because of the variability of serum drug concentrations, pain caused by the injection and its side effect. Therefore, MMWT can be alternative choice for pain relief and control infection of wound instead of drugs, greater patient satisfaction and superior analgesia, prevent inflammation in cesarean section incision wound and help to reduce the side effect of intravenous/intramuscular analgesic drug on human body. Although statistically insignificant result was observed in day 1 and day 2 for pain evaluation, generally they used to respond reduced pain after MMWT. In postoperative day three, we obtained significant pain reduction after exposure to MMWT compared to control group. Tshykevich et al (1998) reported postoperative pain relief after 3-5 session followed by improved cognitive brain function, although these reports were explained in 42 cases study without control group. ${ }^{14}$ The clinical improvement was obtained by reduction of seizure episode with normalization of the bioelectric brain activity and disappearance of the pathologically slow $\square$-waves monitored by means of electroencephalography in epilepsy patients. In our study $54.4 \%$ women could not sleep well because of postoperative pain at lower abdomen and new born baby cry out for milk or passing of urine and stool at night.

While discussing its effect on pain reduction we believe that the wave produced by MMW'T exposed directly over incision site are absorbed by skin's free nerve endings that are spread into epidermis. ${ }^{15}$ After initial exposure to skin, immunocompetent cells such as Langerhans cells and keratinocytes get stimulation to release systemic cytokines. This light is converted into energy and transmitted through the cutaneous nerve through the dorsal root ganglion into the spinal cord. At the first synapse in the spinal cord, there is a release of endogenous opioids. Opioids work by binding to opioids receptor, which are found principally in the central and peripheral nervous system and gastrointestinal tract. The release of endogenous opioids into the blood stream spreads these chemicals throughout the body, and opioids receptors certainly act adequately to relief pain after exposure to millimeter wave therapy. 
Experimented on animal rabbit model, ${ }^{16}$ successfully treated Staphylococcus aureus infected wound with MMWT. A randomized patients-researcher blinded clinical trial of the effects of MMWT (applied for $30 \mathrm{~min}$ for 7 consecutive days) for wound treatment in patient's postoperative abdominal surgery, faster wound healing and decrease pain was achieved in study group compared to control. The intensity of postoperative pain, measured on the numerical rating scale NRS-4 decreased on the third postoperative day in $>90 \%$ of the study group compared to $25 \%$ in control within the same study period ${ }^{17}$, consistently we did obtained reduced pain in our study group. It is understandable that nerves are involved in the reception of these electromagnetic signal neural pathways for transmission of energy to the corresponding regulatory centers in the body. Appearance of electroencephalogram (EEG) changes in children with cerebral palsy ${ }^{18}$ indicate that the central nervous system participates in response to MMWT stimuli. Our current findings that MMWT for pain relief in an area of the body from the exposure site also suggest involvement of a certain central mechanism of transmitting the low-power electromagnetic signal and reacting to it.

\section{CONCLUSIONS}

In summary, MMWT has dual function; firstly it can promote postoperative wound healing by preventing inflammation and infection; secondly it can reduce pain by stimulating opioids. This simple model can reduce administration of oral or intravenous analgesia and antibiotics. It is non invasive, cost effective, non heating, and has no side effect, which is promising modality for clinical therapy and could be the best alternative to pharmaceutical drugs therapy. Our case control study observed significant suppression of pain intensity in postoperative pain after exposure to MMWT. This method can potentially be used as a supplementary or alternative treatment for pain relief. These observations have been confirmed by rapid pain reduction as well as prevention of wound infection by exposure to MMWT within $24 \mathrm{~h}$ of cesarean section. We would like to recommend this safe, simple, and easy therapeutic treatment device for either routine or on demand pain suppression model as well as to cure or prevent incision site infection and inflammation for obstetric cesarean wound management.

\section{Abbreviations}

MMWT - Millimeter Wave Therapy

NRS - Numerical rating scale

EEG - Electroencephalogram

PCI - Post cesarean infection
WBC - White blood cell

L - Lymphocyte

N - Neutrophil

\section{REFERENCES}

1. Rojavin MA and Ziskin MC. Medical application of millimeter waves. QJM 1998; 91: 57-66.

2. Radzievsky AA, Mikhail A. Rojavin, Alan Cowna and Marvin C Ziskin. Suppression of pain sensation caused by Millimeter Waves: A Double-Blinded, Cross-over, prospective human volunteer study. Anesth analg 1999; 88:836-840.

3. Alekseev SI and Ziskin MC. Influence of blood flow and millimeter wave exposure on skin temperature in different thermal models. Bioelectromagnetic 2009; 30: 52-58.

4. Kaplan NM, Smadi AA, Al-Taani MI and El-Qudah MA. Microbiology of wound infection after caesarean section in a Jordanian hospital. East Mediterr Health J 2003; 9: 1068-1074.

5. Adaskevich VP. Clinical efficiency and immunoregulatingneurohumoral effects of millimeter-wave and microwave therapies of atopic dermaitits. Volny $\vee$ Biologii Meditsine 1995; 6: 30-38. [in Russian].

6. Radzievysky AA, Gordiienko OV, Alekseev S, Szabo I, Cowan A and Ziskin MC. Electromagnetic millimeter wave induced hypoalgesia: frequency dependence and involvement of endogenous opioids. Bioelectromagnetics 2008; 29: 284-295.

7. Bone RC, Balk RA, Cerra FB, Dellinger RP, Fein Am, Knaus WA, et al. Definition for sepsis and organ failure and guidelines for the use of innovative therapies in sepsis. The ACCP/SCCM Consensus Conference Committee. American college of chest physician/Society of Critical Care Medicine. Chest 1992; 101:1644-1655.

8. Radziesvky AA, Rojavin MA, Cowan A, Alekseev SI and Ziskin MC. Hypoanalgesic effect of millimeter waves in mice: Dependence on the site of exposure. Life Sci 2000; 66: 2101-2111.

9. Gapeyev $A B$ and Chemeris NK. Effect of continuous and modulated extremely high-frequency electromagnetic radiation on animal cells. Review. Part III. Biological effect of continuous EHF EMR. Herald Mod Med Techol 2000; 7: 20-25. [in Russian].

10. Gapeyev AB, Mikhailik EN and Chemeris NK. Antiinflammatory effects of low-intensity extremely high frequency electromagnetic radiation: Frequency and power dependence. Bioelectromagnetics 2008; 29: 197-206.

11. Logani M, Anga A, Szabo I, Agelan A, Irizarry A and Ziskin M. Effect of millimeter waves on cyclophosphamide induced suppression of the immune system. Bioelectromagnetics 2002; 23:614-621.

12. Schaffer $M$ and Barbul $A$. Lymphocytes function in wound healing and following injury. Br J surg 1998; 85: 444-460.

13. Makar V, Logani M, Szabo I and Ziskin M. Effect of millimeter waves on cyclophosphamide-induced suppression of $\mathrm{T}$ cell functions. Bioelectromagnetics 2003; 24: 356-365.

14. Tsyhkevich TG, Bersnev VP and Stepanova TS. The use of millimeter waves in neurosurgery under electrophysiological control. Vopr Kurortol Fizioter Lech Fiz Kult 1998; 57: 667-671.

15. Usichenko TI, Edinger H, Gizhko VV, Lehmann C, Wendt M and Feyerherd F. Low-intensity electromagnetic millimeter waves for pain therapy. Evid Based Complement Alternat Med 2006; 3: 201-207.

16. Korpan NN, Resch KL and Kokoschinegg P. Continuous microwave enhances the healing process of septic and aseptic wounds in rabbits. J Surg Res 1994; 57: 667-671. 
17. Korpan NN and Saradeth T. Clinical effects of continuous microwave for postoperative septic wound treatment: A double-blind controlled trial. Am J Surg 1995; 170: 271-276.
18. Lebedeva NN. Neurophysiological mechanism of low intensity electromagnetic field biological mechanisms of low intensity electromagnetic field biological effects. Radiotekhnika 1997; 4:62-66.

Authors Contribution:

PM - design the study, performed the clinical therapy, analysed the data, drafted the manuscript \& reviewed the manuscript. SY - Contributed the study design; LYP - Reviewed the manuscript.

Source of Support: Nil, Conflict of Interest: None declared. 